\title{
EXPERIMENTAL STUDY OF PROTON-BEAM HALO INDUCED BY BEAM MISMATCH IN LEDA *
}

\author{
T.P.Wangler, C.K.Allen, K.C.D.Chan, P.L. Colestock, K.R.Crandall ${ }^{\mathrm{a}}$, R.W.Garnett, D.Gilpatrick, \\ W. Lysenko, J. Qiang, J.D.Schneider, M.E.Schulze ${ }^{\mathrm{b}}$, R.L.Sheffield, and H.V.Smith \\ Los Alamos National Laboratory, Los Alamos, NM 87544 \\ ${ }^{\mathrm{a}}$ TechSource, Santa Fe, NM 87594-1057 \\ ${ }^{\mathrm{b}}$ General Atomics, Los Alamos, NM 87545 \\ *Work supported by US Department of Energy
}

\begin{abstract}
We report measurements of transverse beam halo in mismatched proton beams in a 52-quadrupole FODO transport channel following the 6.7-MeV LEDA RFQ. Beam profiles in both transverse planes are measured using beam-profile diagnostic devices that consist of a movable carbon filament for measurement of the dense beam core, and scraper plates for measurement of the halo. The gradients of the first four quadrupoles can be independently adjusted to mismatch the RFQ output beam into the beam-transport channel.
\end{abstract}

\section{INTRODUCTION}

As a result of theoretical research during the past decade, we believe space-charge forces in mismatched beams are the main source of beam halo in high current proton beams. A beam is matched when there is a balance between the inwardly directed focusing force and the outwardly directed space-charge force plus a pressure force from the emittance. Beam mismatch corresponds to an imbalance in the forces, which acts in two ways. First, mismatch produces an immediate increase in particle transverse amplitudes and coherent oscillations of the rms beam sizes. Second, the nonlinear space-charge force seen by individual particles, acting while the beam is undergoing coherent rms-size oscillations, slowly drives some particles to even larger amplitudes; this mechanism has been described by a particle-core model $[1,2,3]$ and identified as a parametric resonance [2]. The experiment will test these ideas by observing the properties of the beam after introducing a deliberate mismatch.

\section{BEAM HALO EXPERIMENT}

A 52-quadrupole FODO beam-transport channel was installed at the end of the Low-Energy Demonstration Accelerator (LEDA) 6.7-MeV, 350-MHz RFQ to carry out a first experimental study of beam-halo formation in a proton beam $[4,5]$. The channel is long enough for the development of about 10 mismatch oscillations, enough to observe the initial stages of emittance growth and halo formation caused by mismatch. A large compliment of beam diagnostics is provided [6]. The key diagnostic component is a special transverse beam profile scanner
[7] consisting of a thin, $33 \mu$ diameter, carbon wire for measurement of the dense beam core, and a pair of 1.5$\mathrm{mm}$ thick scraper plates to provide greater sensitivity for measurement of the outer halo regions. Each wire and its associated pair of scraper plates are mounted on a common movable frame. As the protons pass through the wire a signal is induced from secondary electron emission. The scraper plates are water cooled and are constructed of graphite brazed onto copper. A signal is induced by the beam protons that stop in the plates. The data from the wires and the plates are combined using computer software to produce a single distribution. The device has a large dynamic intensity range of about $10^{5}: 1$. Nine measurement stations, shown in Fig. 1, are located midway between pairs of quadrupoles; at each location both the horizontal and vertical projected distributions are measured. The first station is located after quadrupole 4 . The next four stations are located after the beam has debunched at quadrupoles $20,22,24$, and 26 . The last four stations are located after quadrupoles $45,47,49$, and 51.

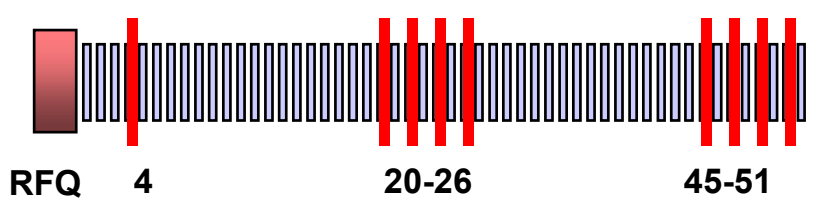

Figure 1: Block diagram of the 52 quadrupole transport channel showing the locations of the 9 profile scanners.

Beam matching was done by adjusting the first four quadrupoles to produce equal rms sizes in both $\mathrm{x}$ and $\mathrm{y}$. We estimate a measurement uncertainty for the rms beam sizes of less than $50 \mu$, caused mostly by transverse beam jitter. A least-squares-fitting procedure was used based on measurements of derivatives of rms sizes with respect to matching quadrupole gradients. The Courant-Snyder parameters of the matched beam can be calculated using TRACE3D. Pure mode mismatches are calculated by scaling the Courant-Snyder parameters to the desired values and finding the settings of the matching quadrupoles that produce these values. For example, multiplying each of the four matched transverse CourantSnyder ellipse parameters $\alpha_{x}, \beta_{x}, \alpha_{y}$, and $\beta_{y}$ by the same scaling factor produces a pure breathing-mode mismatch. 

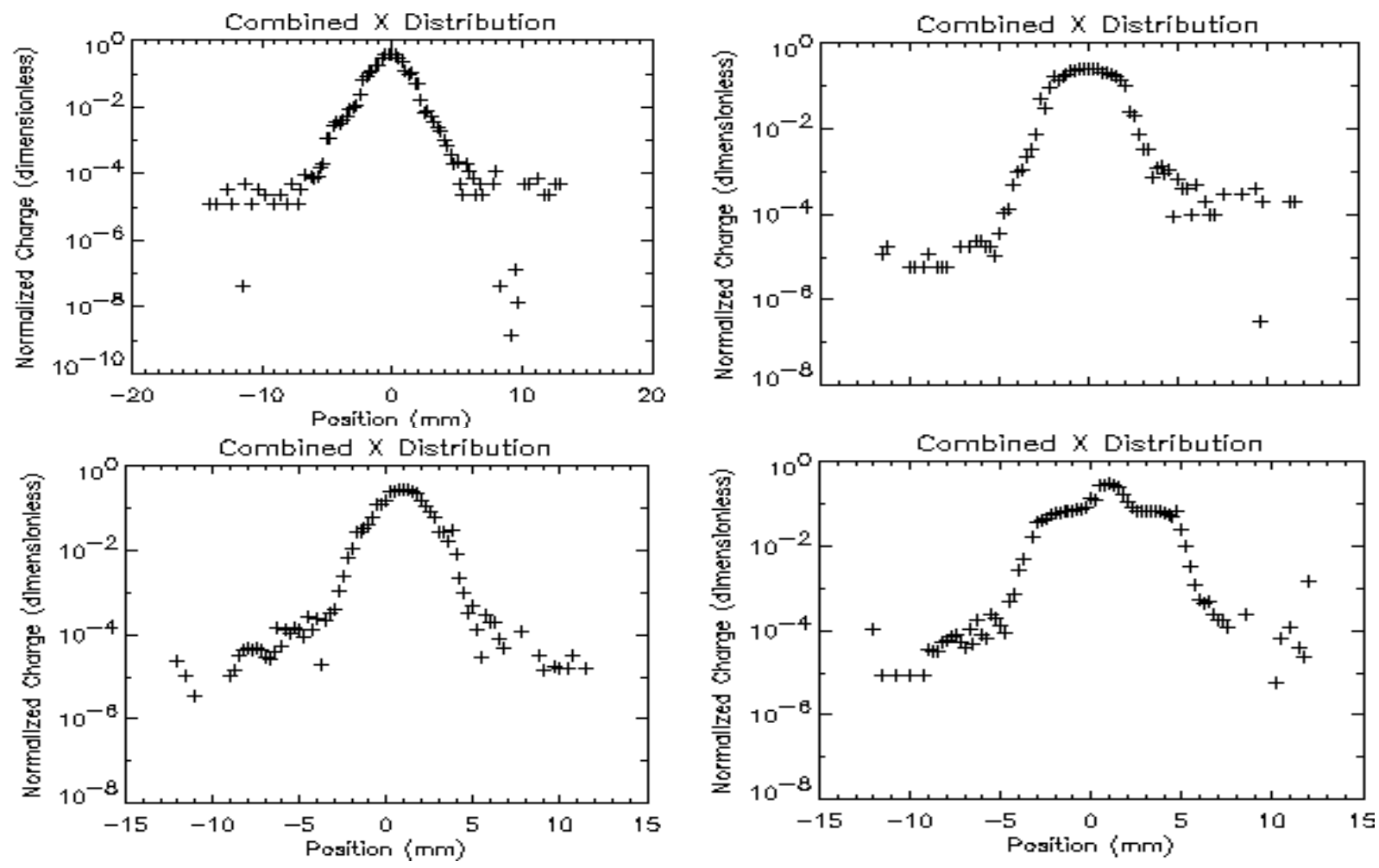

Figure 2: Measured beam profiles in $\mathrm{x}$ at $75 \mathrm{~mA}$ : (upper left) scanner 22, $\mu=1.0$, matched, (upper right) scanner 22, $\mu=1.5$ mismatched, (lower left) scanner $51, \mu=1.0$ matched, (lower right) scanner $51, \mu=1.5$ mismatched.

The mismatch strength is measured by a parameter $\mu$, which equals the ratio of the rms size of the mismatched beam to that of the matched beam.

The beam from the ion source is pulsed at $1 \mathrm{~Hz}$ to allow the use of interceptive beam diagnostics. The measurement cycle consists of the following steps. The cw RFQ is de-energized with an RF blanking pulse, and the ion source is turned on. As the $75-\mathrm{keV}$ dc injector beam current rises, the beam is injected into the unpowered RFQ. After about $2 \mathrm{msec}$ the RF blanking pulse is removed; the RFQ fields approach a steady state after another $5 \mu \mathrm{sec}$. The ion source and RF fields remain on for another $30 \mu \mathrm{s}$ for data acquisition. The beamprofile diagnostics are in a fixed position, so only one wire or scraper is in the beam at a time; all other wires and scrapers are out of the beam-pipe aperture. The wire or scraper in the beam accumulates beam-induced charge over about $30 \mu \mathrm{sec}$. Only the charge collected over the last $10 \mu \mathrm{sec}$, when the beam has reached a steady state, is selected for the recorded data. Then the dc injector is turned off, and the beam profile scanners are repositioned for the next cycle.

Among the methods used to characterize the beam are: 1) the rms emittance, 2) a dimensionless shape parameter called the kurtosis, and 3) the maximum detectable amplitude. A least squares procedure is used to calculate rms emittances at the RFQ exit from rms size measurements at scanner 4 using 17 different settings of the first four matching quadrupoles [5]. Another variant of the method, based on FWHM values, is used in Ref. [5]. Rms emittances at each of the two scanner clusters are calculated from a least-squares procedure using rms size measurements from the profiles at the four scanners in each cluster. The kurtosis is a function of the second and fourth moments of the distribution [8]. Defined as $\mathrm{h}=\left\langle\mathrm{x}^{4}>\right|\left\langle\mathrm{x}^{2}\right\rangle^{2}-2$, it is equal to 0 for a $\mathrm{K}-\mathrm{V}$ distribution, and 1 for a Gaussian profile. A Gaussian profile with added tails from halo growth results in $\mathrm{h}>1$, making it an attractive measure of halo growth. But, if mismatch causes the beam profile to approach a more rectangular shape, $\mathrm{h}$ can decrease. Other measures of the halo are discussed in Ref. [4]. The maximum detectable amplitude provides a lower limit to the extent of the beam, and is calculated from the intersection of the transverse beam profile with the background noise level. The intersection is determined from a statistical signal-to-noise parameter calculated for each data point.

\section{INITIAL RESULTS FOR 75 MA}

Measurements have been made at 16, 50, and $75 \mathrm{~mA}$. In this paper we report the results at $75 \mathrm{~mA}$. First, the measured transverse profiles (see Fig.2) show unexpected structure in the form of shoulders in the semilog plots, and some asymmetric profiles at $10^{-3}$ level. The shoulders and asymmetries are most prominent for the mismatched beams. Figures 3 and 4 show the $\mathrm{x}$ and $\mathrm{y}$ rms sizes at the different scanners for a matched and mismatched case, respectively. The rms emittances, shown in Fig. 5, grow along the channel with a rate that increases with increasing mismatch strength. The kurtosis (not shown) generally decreases with increasing mismatch strength. This decrease can be qualitatively explained by the development of the shoulders, which makes the mismatched beam profiles more rectangular as the 
mismatch strength is increased. The decrease dominates over the kurtosis increase expected from tail formation during halo growth. The maximum detectable amplitude,

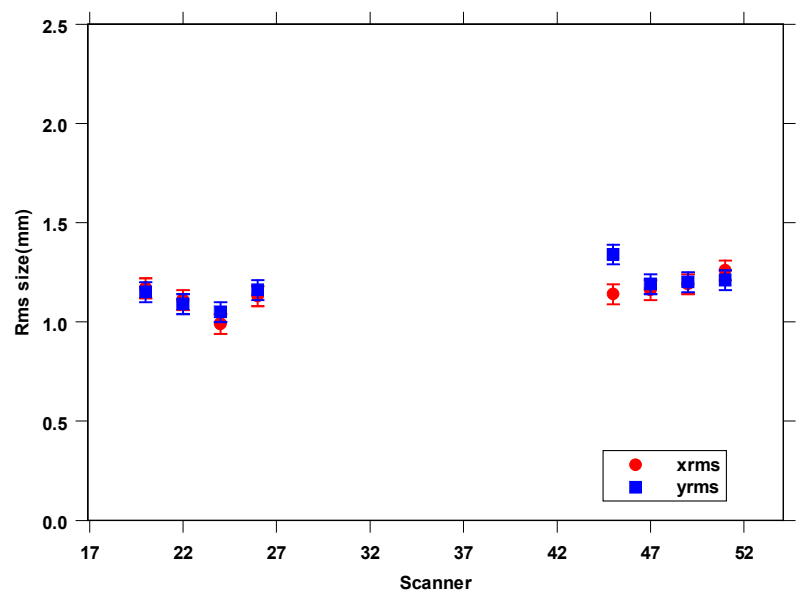

Figure 3: Rms displacements at the 8 profile scanners for matched beam case $(\mu=1.0)$ at $75 \mathrm{~mA}$.

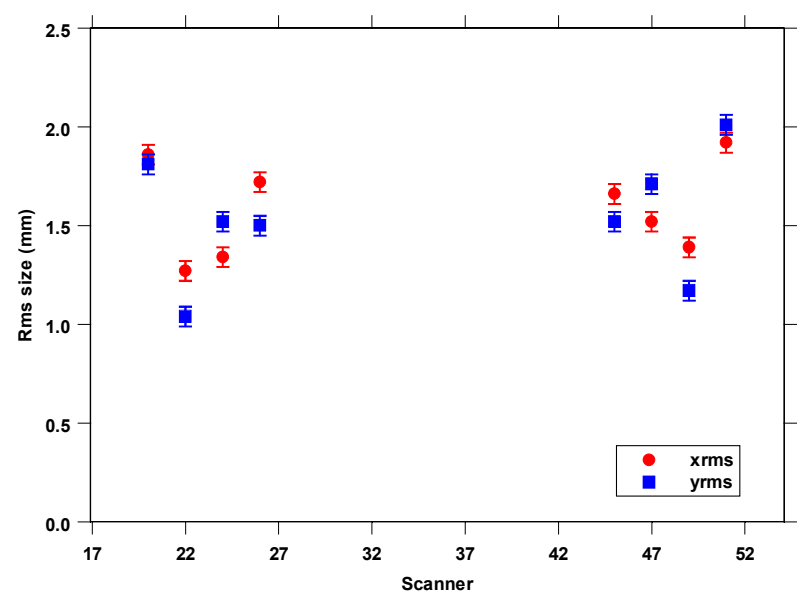

Figure 4: Rms displacements at the 8 profile scanners for the breathing mode mismatch case $(\mu=1.5)$ at $75 \mathrm{~mA}$.

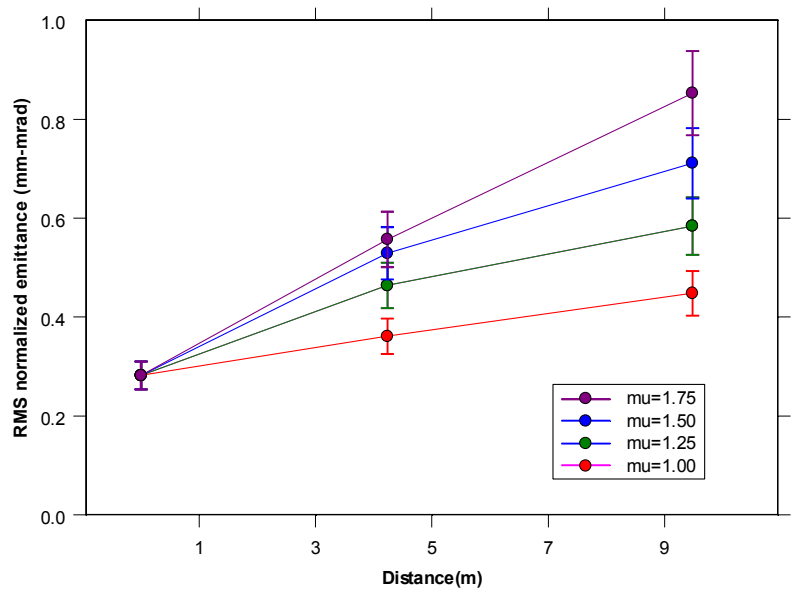

Figure 5. Rms normalized emittance in $\mathrm{x}$ at $75 \mathrm{~mA}$ versus distance at different breathing mode mismatch strengths. about 6 to $8 \mathrm{~mm}$, is approximately what was expected from multiparticle simulations.

\section{MULTIPARTICLE SIMULATIONS}

Multiparticle simulations in the transport channel have been carried out using the IMPACT code with a 3D particle-in-cell space-charge subroutine. The simulations use 10-million particles and an input beam distribution determined from multiparticle simulation through the RFQ, using previous emittance measurements made at the location of the RFQ entrance. The measured results are not in good agreement with these simulations. More work is required to investigate the dependence of the simulation results on the input beam characteristics.

\section{SUMMARY}

The measurements at $75 \mathrm{~mA}$ show rms emittance growth increasing with increasing beam mismatch, which is qualitatively consistent with our expectations for spacecharge forces acting in a mismatched beam. The maximum extent of the halo is consistent with predictions from numerical simulation. But, other measured results are not in good agreement with the simulations. The shapes of the transverse beam profiles are not understood, and the measured transverse rms emittances grow more rapidly then predicted. Preliminary results suggest that halo is present in the beam emerging from the RFQ. One hypothesis to explain some of these observations is that the RFQ output beam may include a few percent of offenergy particles. However, initial measurements show no direct evidence for off-energy particles. Work is in progress towards a better understanding of these measurements.

\section{REFERENCES}

1 J.S. O'Connell, T.P.Wangler, R.S.Mills, and K.R.Crandall, Proc. of 1993 Part. Accel. Conf., IEEE Catalog No. CH3279-7, 3657-3659.

2 R.L. Gluckstern, Phys. Rev.Lett. 73, 1247 (1994). 3 For an overview, see T.P. Wangler, K.R. Crandall, R. Ryne, and T.S. Wang, Phys.Rev. ST Accel. Beams 1 (084201)1998.

4 P.L. Colestock, et. al, "Measurements of Halo Generation for a Proton Beam in a FODO Channel," these proceedings.

5 M.E. Schulze, et al, "Characterization of the Proton Beam from the 6.7 MeV LEDA RFQ," these proceedings. 6 J.D. Gilpatrick, et al., "Experience with the Low Energy Demonstration Accelerator (LEDA) Halo Experiment Beam Instrumentation," these proceedings.

7 J.D. Gilpatrick, et al., "Beam-Profile Instrumentation for Beam Halo Experiment: Overall Description and Operation," these proceedings.

8 C.K. Allen and T.P. Wangler, "Parameters for Quantifying Beam Halo", these proceedings. 